Anjou. Maine. Poitou-Charente. Touraine

\title{
Charles de Blois et Jeanne de Penthièvre, duc et duchesse de Bretagne et leur vicomté de Limoges
}

L'évidence des comptes

Erika Graham-Goering et Michael Jones

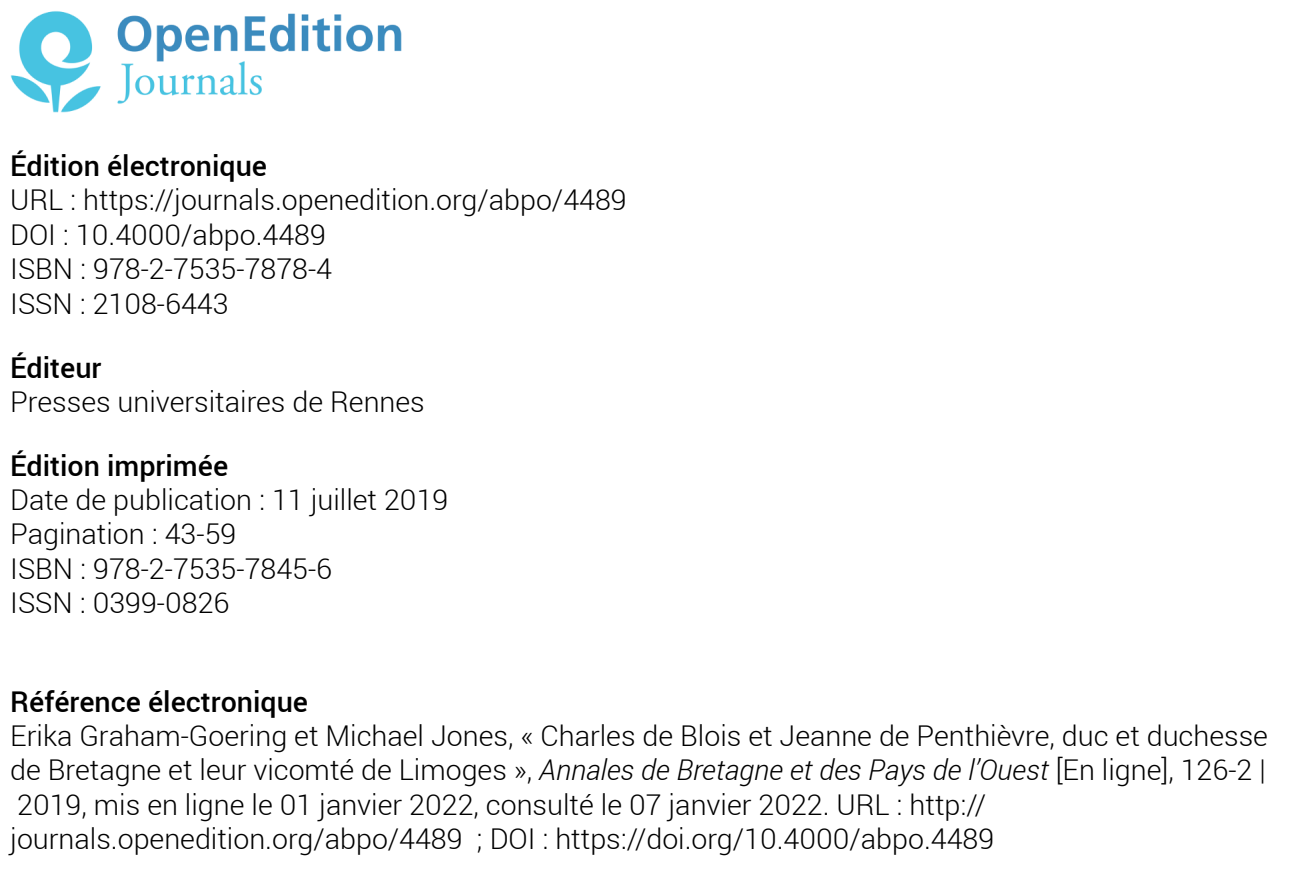

(C) Presses universitaires de Rennes 


\title{
Charles de Blois et Jeanne de Penthièvre, duc et duchesse de Bretagne et leur vicomté de Limoges
}

\section{L'évidence des comptes ${ }^{1}$}

\author{
Erika GRAHAM-GOERING \\ Chercheuse postdoctorale en histoire médiévale française, \\ université de Gand \\ Michael JONES \\ Professeur émérite d'histoire médiévale française, \\ université de Nottingham
}

Le mariage d'Arthur, petit-fils de Jean $\mathrm{I}^{\mathrm{er}}$, duc de Bretagne, avec Marie, unique héritière de Gui VI et Marguerite de Bourgogne, vicomte et vicomtesse de Limoges, le 22 juillet 1275, fit entrer la vicomté dans le domaine des ducs de Bretagne ${ }^{2}$. Arthur, âgé de tout juste 13 ans au moment de son mariage (Marie a alors 15 ans), passa ensuite beaucoup du temps à

1. Depuis le colloque de Brest et la soumission de ce texte, nous avons traité le contexte de la succession de Charles de Blois et Jeanne de Penthièvre à la vicomté de Limoges en plus de détails dans Graham-Goering, Erika, Jones, Michael et Yeurc'H, Bertrand, Aux origines de la guerre de succession de Bretagne. Documents (1341-1342), Rennes, Presses universitaires de Rennes, Sources médiévales de l'histoire de Bretagne, nº 9, 2019, et dans Graham-Goering, Erika, Princely Power in Late Medieval France: Jeanne de Penthièvre and the War for Brittany, Cambridge, Cambridge University Press (à paraître), une version remaniée de la thèse doctorale citée ci-dessous, note 32. Erika Graham-Goering tient à remercier l'appui financier accordé à ses recherches par l'université York et par le ERC Starting Grant no. 677502: STATE - Lordship and the Rise of States in Western Europe, 13001600 (PI: Frederik Buylaert, Ghent University; co-PI: Justine Firnhaber-Baker, University of St Andrews).

2. Roblin, Vincent, Recueil des actes des vicomtes de Limoges ( $X^{e}$-XIV siècle), Genève, Librairie Droz, 2009, p. 41-42. Malheureusement, bien qu'il ait amassé beaucoup de détails Roussel, Claude Youenn, La mainmise des Bretons sur le Limousin-Périgord 12741522. Noblesse et troupes bretonnes en Limousin, Occitanie, Espagne, Italie, Grèce, Paris, Guénégaud, 2002, manque de rigueur scientifique (voir le compte rendu par JonES, Michael, dans Mémoires de la Société d'histoire et d'archéologie de Bretagne, t. LXXXIV, 2006, p. 495-496). 
Limoges avant son avènement comme duc en 1305, tout comme le fera également son propre fils, le futur Jean III (1312-1341), apprenant le métier de prince, assisté par quelques Bretons qui occupèrent plus tard des positions importantes dans l'administration auprès du duc. Après une courte période (1314-1317) au cours de laquelle Guy, frère puîné de Jean III, posséda la vicomté comme apanage ${ }^{3}$, Jean III exerça le gouvernement de la vicomté pour le reste de son règne. Cette période fut rappelée plus tard avec affection par quelques-uns de ses sujets Limougeauds, tels les consuls et habitants de la châtellenie de Limoges, qui, vers 1350, se souvenaient encore du gouvernement du " bon duc Jehan ${ }^{4}$ ", bien que l'évidence documentaire qui fonde une telle assertion soit pour nous assez maigre ${ }^{5}$. De fait, la plupart des documents concernent des affaires financières : une quittance pour 1000 livres reçues des habitants de Limoges lors de son adoubement comme chevalier en $1308^{6}$, la nomination de monnayeurs à Nantes et à Limoges le 30 mai $1340^{7}$, et la mention du trésor, comprenant une somme assez importante d'argent frappé à Limoges (1980 livres monnaie de Limoges sur un total de 6045 livres 15 sous), trouvé par les exécuteurs du testament du duc Jean III dans la sacristie de la cathédrale SaintPierre de Nantes en juin $1341^{8}$, mention qui est peut-être à l'origine du récit de Froissart de la saisie prétendue par Jean de Montfort du trésor du feu duc Jean III à Limoges ${ }^{9}$. Ensuite, Charles de Blois et Jeanne de Penthièvre levèrent des sommes d'argent assez importantes sur leur vicomté. En cela, ils continuaient une politique bien établie par les ducs qui furent leurs pré-

3. Robuin, Vincent, Recueil des actes..., op. cit., p. 44.

4. JonEs, Michael, Recueil des actes de Charles de Blois et Jeanne de Penthièvre, duc et duchesse de Bretagne (1341-1364), suivi des actes de Jeanne de Penthièvre (1364-1381), Rennes, Presses universitaires de Rennes, 1996, p. 273; cette édition, avec un supplément, a été numérisée : http://books.openedition.org/pur/28420

5. Très peu de mandements ou de lettres du duc concernant l'administration de sa vicomté ont survécu. Philippe VI a confirmé deux lettres de Jean III comme vicomte en juillet 1331 (Arch. nat., JJ 66, fo 319, nº 768 et 769. Voir VIARD, Jules, VALLÉE, Aline et FAVIER, Jean, Registres du trésor des chartes, Inventaire analytique, Règne de Philippe de Valois. Première partie JJ $65^{A}$ à 69, et Registres... Deuxième partie. JJ 70 à 75, Paris 1978-1979 (désormais Registres I et Registres II), Registres I, $\mathrm{n}^{\text {os }} 1407$ et 1408) et il y a un vidimus des lettres du duc pour Limoges du $1^{\text {er }}$ septembre 1339 (Arch. nat., JJ 74 f f $^{\circ} 206 \mathrm{v}$ n $^{\circ} 362$, Registres II, $\mathrm{n}^{\circ}$ 5182). Pour une protestation de Jean comme vicomte contre tentatives royales pour forcer les habitants de la vicomté à servir en armes contre les Anglais en 1324 : BnF, collection Doat, ms 243, fo 14-20.

6. RobLin, Vincent, Recueil des actes..., op. cit., p. 344, nº 193.

7. Archives historiques du Limousin, t. IX, 1904, p. 167-169, $\mathrm{n}^{\circ}$ XXXII.

8. Jones, Michael et Charon, Philippe, Comptes du duché de Bretagne. Les comptes, inventaires et exécution des testaments ducaux, 1262-1352, Rennes, Presses universitaires de Rennes/Société d'histoire et d'archéologie de Bretagne, 2017, p. 397-399.

9. Jones, Michael, "The Breton Civil War ", dans Palmer, J. J. N. (dir.), Froissart : Historian, Woodbridge, Boydell and Brewer, 1981, p. 64-88 et 169-172 [réimp. dans JonES, Michael, The Creation of Brittany, Londres, Hambledon Press, 1988, p. 197-218], pour une vue sceptique du récit de Froissart sur les événements en Bretagne en 1341, position que les travaux ultérieurs ont simplement renforcée (cf. JonEs, Michael, " Ancenis, Froissart et le début de la guerre de succession en Bretagne (1341) ", Mémoires de la Société d'histoire et d'archéologie de Bretagne, t. 77, 1999, p. 91-102). 
décesseurs, exigeant un maximum de revenu du Limousin, bien que cette région fût beaucoup moins riche que la Bretagne ${ }^{10}$.

Avant sa mort, survenue le 30 avril 1341, Jean III a assigné à sa troisième épouse, Jeanne de Savoie, un douaire sur sa seigneurie vicomtale ${ }^{11}$. Mais cette possession n'était que viagère, aussi il y eut, comme pour le duché de Bretagne, une question de droit à résoudre : à qui revenait la vicomté? En effet, chacun des prétendants au duché de Bretagne affirma son droit sur la vicomté, alors que la sœur aînée de Jean de Montfort, Jeanne de Bretagne, dame de Cassel, faisait valoir des créances ${ }^{12}$. Par la suite, bien que l'arrêt de Conflans (7 septembre 1341) ait autorisé Charles de Blois à faire hommage pour le duché de Bretagne, au nom de sa femme Jeanne de Penthièvre, comme successeur de Jean III ${ }^{13}$, le couple ne put entrer immédiatement en possession de la vicomté, la duchesse douairière Jeanne étant toujours en vie et ce jusqu'en $1344^{14}$. La mort de Jeanne de Savoie fit que Charles de Blois ainsi que Jean de Montfort firent valoir leurs droits sur la vicomté devant le parlement de Paris qui, par un arrêt du 10 janvier 1345, rejeta " les prétensions émises par Jean de Montfort " et autorisa Charles de Blois « à prêter hommage au roi de France pour la dite vicomté " comme

10. TRICARD, Jean, Les campagnes limousines du XIV ${ }^{\mathrm{e}}$ au XVI ${ }^{\mathrm{e}}$ siècle. Originalité et limites d'une reconstruction rurale, Paris, Publications de la Sorbonne, 1996.

11. Jones, Michael, Recueil des actes de Charles de Blois..., op. cit., $\mathrm{n}^{\circ} 22$ pour les droits de Jeanne de Savoie sur Limoges. Auparavant, la deuxième femme de Jean III a également reçu son douaire sur la vicomté (voir Arch. nat., JJ 66, nº 212 et SPINOSI, Caroline, « Un règlement pacifique dans la succession de Jean III, duc de Bretagne à la vicomté de Limoges ", Revue historique du droit français et étranger, $4^{\mathrm{e}}$ série, t. XxxIX, 1961, p. 455).

12. SPINOSI, Caroline, "Un règlement pacifique... ", art. cit., p. 453-467. Pour une protestation par Jeanne de Montfort, dame de Cassel, au sujet des ses droits sur la vicomté de Limoges, voir Arch. dép. du Nord, B 818, n 7401, 24 août 1341, lettres de Philippe VI, publié dans Jones, Michael, "Some documents relating to the disputed succession to the duchy of Brittany, 1341 ", Camden Miscellany, t. xxIv, 1972 [Londres, Royal Historical Society, Camden Fourth Series, Volume 9], p. 70-71.

13. Duchesne, André, Histoire de la maison de Châtillon, Preuves, Paris, Sébastien Cramoisy, 1621, p. 122-125; LoBInEAU, Gui Alexis, Histoire de Bretagne, Paris, la V ve François Muguet, 1707, t. II, col. 485-488, réimprimé dans MoRICE, Pierre Hyacinthe, Mémoires pour servir de preuves à l'histoire ecclésiastique et civile de Bretagne, Paris, C. Osmont, 17421746, t. I, col. 1442-1447 pour trois éditions anciennes de l'arrêt (dont la copie enregistrée contemporaine utilisée par Duchesne se trouve dans Arch. nat., $\mathrm{X}^{1 \mathrm{~A}} 10 \mathrm{f}^{\mathrm{o}} 231$, tandis que la version consultée par Lobineau et Morice est un vidimus de 1361, maintenant Arch. dép. Loire-Atlantique, E 165, n ${ }^{\circ} 3$ ). Une édition moderne d'après une autre copie contemporaine (Arch. nat., $\mathrm{X}^{1 \mathrm{~A}} 9$ fo $201 \mathrm{v}$ ), fut faite par Pierre Clément Timbal et Josette Metman (Timbal, Pierre-Clément et Metman, Josette, Études d'histoire du droit international privé, Paris, Éditions du Centre national de la recherche scientifique, 1967, p. 116-119; une traduction de MEIJERs, Eduard Maurits, Bijdrage tot de Geschiedenis van het international privaaten strafrecht in Frankrijk en de Nederlanden, La Haye, 1914).

14. Contrairement à Jean de Montfort, Jeanne et Charles avaient réclamé la succession de Limoges en même temps que celle de Bretagne : BnF, ms fr. 18697, fo 135-135v (document ignoré par SPINOSI, Caroline, « Un règlement pacifique... ", art. cit., p. 456, ce qui rend sa chronologie de la dispute inexacte). 
il l'avait déjà fait pour la Bretagne ${ }^{15}$. Sans attendre cet arrêt, Charles de Blois avait incorporé dans sa titulature le titre de "vicomte de Limoges " qui figure dans des lettres données " en noz tentes devant Guerande " le 8 août $1344^{16}$. Divers indices suggèrent qu'il faut placer entre décembre 1343 et février 1344 le moment où Charles et Jeanne établirent leur administration sur la vicomté ${ }^{17}$. Les fragments de comptes, qui sont le sujet principal de cette communication, tels ceux de Pierre Molin le jeune, receveur du duc de Bretagne dans la vicomté de Limoges, l'attestent : ils commencent en 1344 et ils se terminent au début de l'année $1347^{18}$. Ainsi, sont-ils probablement les premiers comptes que cet officier ait préparés. Ils sont complétés par d'autres fragments de comptes d'Aymeri de Bonneval, receveur ou prévôt de la châtellenie de Ségur[-le-Château] pour les années 1345-1346 ${ }^{19}$. Ces comptes semblent être non seulement les premiers comptes vicomtaux mais aussi les premiers comptes seigneuriaux du Limousin. Siméon Luce les a cités brièvement dans sa remarquable Histoire de Bertrand du Guesclin et de son époque $(1876)^{20}$, mais ils ont été presque ignorés par des historiens plus récents bien qu'ils donnent des informations intéressantes sur l'histoire sociale, économique et politique de la vicomté ainsi que sur son administration. Ils éclairent aussi une période difficile durant laquelle la vicomté a été affectée par les premières dévastations en lien avec la guerre de Cent Ans, génératrices, pour les décennies suivantes, d'une dislocation sociale et économique dont les effets se font sentir jusqu'à la fin du $\mathrm{XV}^{\mathrm{e}}$ siècle ainsi que l'a montré Jean Tricard ${ }^{21}$.

Avant de nous concentrer sur les comptes, évoquons en quelques mots la structure administrative de la vicomté et l'économie locale du Limousin au début du XIve siècle. Située au sud et à l'est des comtés de la Marche, d'Angoulême et de Périgord, limitée au sud par les vicomtés de Turenne et de Comborn, par l'Auvergne à l'est, la vicomté se composait de plu-

15. SPINOSI, Caroline, "Un règlement pacifique... ", art. cit., p. $453-467$ (p. 453 pour la citation); Charles de Blois reçut la permission de rendre hommage pour la vicomté le 10 janvier 1345 (Jones, Michael, Recueil des actes de Charles de Blois..., op. cit., n ${ }^{\circ} 55$ ).

16. Ibidem, $\mathrm{n}^{\circ} 30$.

17. Ibid., n ${ }^{\circ} 22,20$ décembre 1343, pour les lettres (ayant la force d'un testament) de Jeanne de Penthièvre laissant la vicomté à Charles de Blois, mais réservant les droits de Jeanne de Savoie. Vers la fin février ou le début mars 1344, ils semblent avoir pris le contrôle de certaines des ressources financières de la vicomté et ont certainement accordé des subventions dans le Limousin avant le 5 juillet 1344 (ibid., $\mathrm{n}^{\circ} 29$ ).

18. Arch. dép. des Pyrénées-Atlantiques, E 624, $n^{\text {os }} 1$ et 2, édités dans JonEs, Michael et Charon, Philippe, Comptes du duché de Bretagne..., op. cit., p. 403-416, où les dates les plus anciennes citées dans les comptes sont en mai et juin 1345.

19. Arch. dép. des Pyrénées-Atlantiques, E 863, édité en annexe ci-dessous.

20. Luce, Siméon, Histoire de Bertrand du Guesclin et de son époque. La jeunesse de Bertrand, Paris, Librairie Hachette et Cie, 1876, p. 511, où il publia trois articles du compte de Molin (voir Jones, Michael et Charon, Philippe, Comptes du duché de Bretagne..., op. cit., p. 413 art. 93, 97 et 101).

21. Pas de mention de ces comptes dans TRICARD, Jean, Les campagnes limousines..., op. cit., bien qu'il donne un bon compte rendu des effets de la guerre et de la peste sur la région. 
Figure 1 - Les châtellenies de la vicomté de Limoges vers 1300

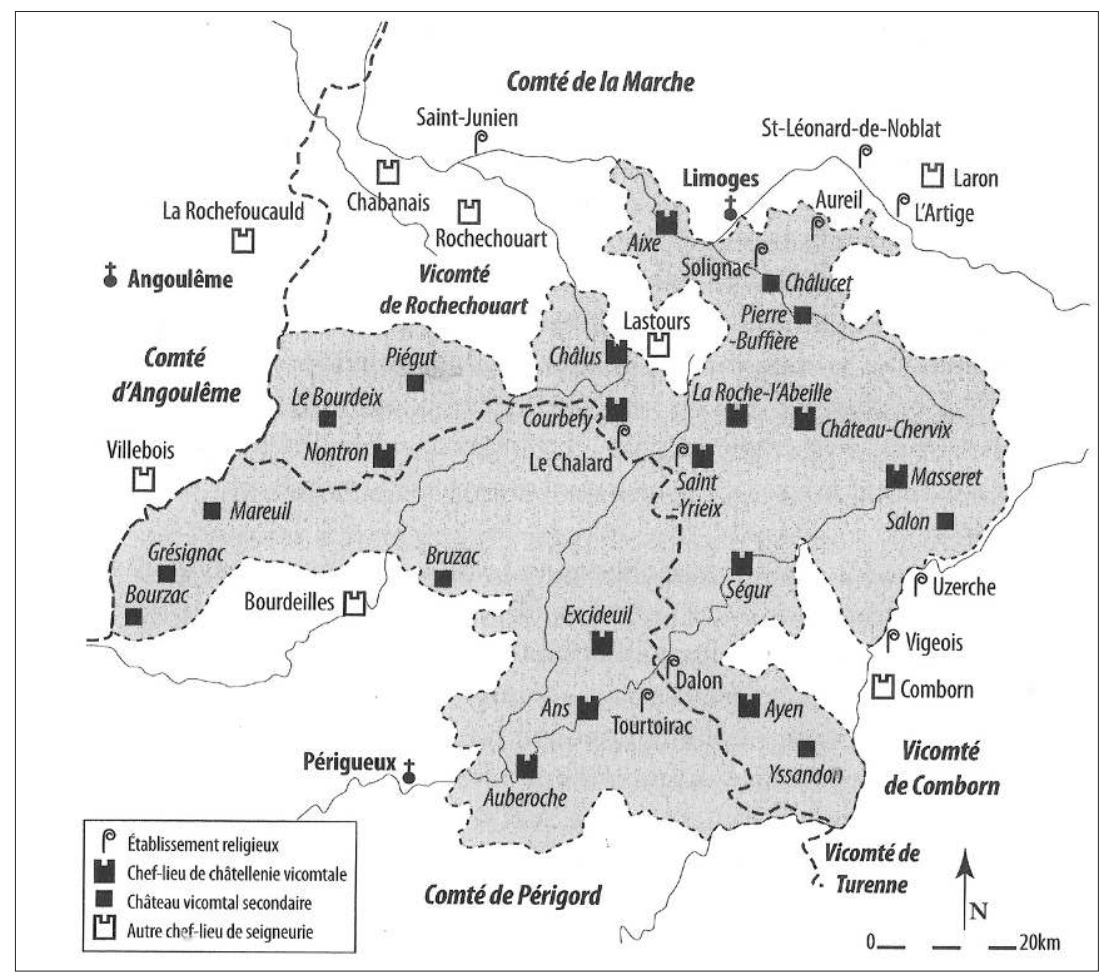

(d'après RoBLIN, Vincent, Recueil des actes..., op. cit., p. 14)

sieurs châtellenies modestes. À la fin du XIII ${ }^{\mathrm{e}}$ siècle, la plupart d'entre elles étaient aux mains du vicomte, mais il était obligé de rendre l'hommage pour quelques-unes à des seigneurs supérieurs comme l'abbé de Solignac (pour Aixe-sur-Vienne ${ }^{22}$ ), l'abbé de Saint-Martial de Limoges (pour une partie de la cité de Limoges elle-même, Pierre-Buffière et Château-Chervix ${ }^{23}$ ), et au chapitre de Saint-Yrieix (pour Ségur ${ }^{24}$ ). Par ailleurs, il partageait la juridiction de la ville de Limoges avec l'évêque, ce qui fut source de débats au long cours. La carte de "La vicomté de Limoges et son environnement (XIII ${ }^{\mathrm{e}}$ siècle) " parue dans l'œuvre inestimable de Vincent Roblin, le Recueil des actes des vicomtes de Limoges, montre l'existence de treize châtellenies vicomtales principales ${ }^{25}$ (fig. 1). Dans nos comptes, des revenus pro-

22. JonEs, Michael, Recueil des actes de Charles de Blois..., op. cit., n ${ }^{\circ} 54$ et 59 pour l'hommage du duc à Pierre de Pompadour, abbé de Solignac pour Aixe, 9 mars 1345.

23. Ibid., nº 266, avant 13 février 1362, demande pour répit d'hommage à l'abbé de SaintMartial, et ibid., $\mathrm{n}^{\circ} 281$, prestation d'hommage, 28 novembre 1363.

24. Ibid., nº 305, 1365, répit d'hommage donné par le doyen et le chapitre de Saint-Yriex à Jeanne pour la seigneurie de Ségur.

25. Roblin, Vincent, Recueil des actes..., op. cit., p. 14, figure 1. 
Figure 2 - Les pertes de feux dans la vicomté de Limoges du $X I V^{e}$ au $X V^{e}$ siècle

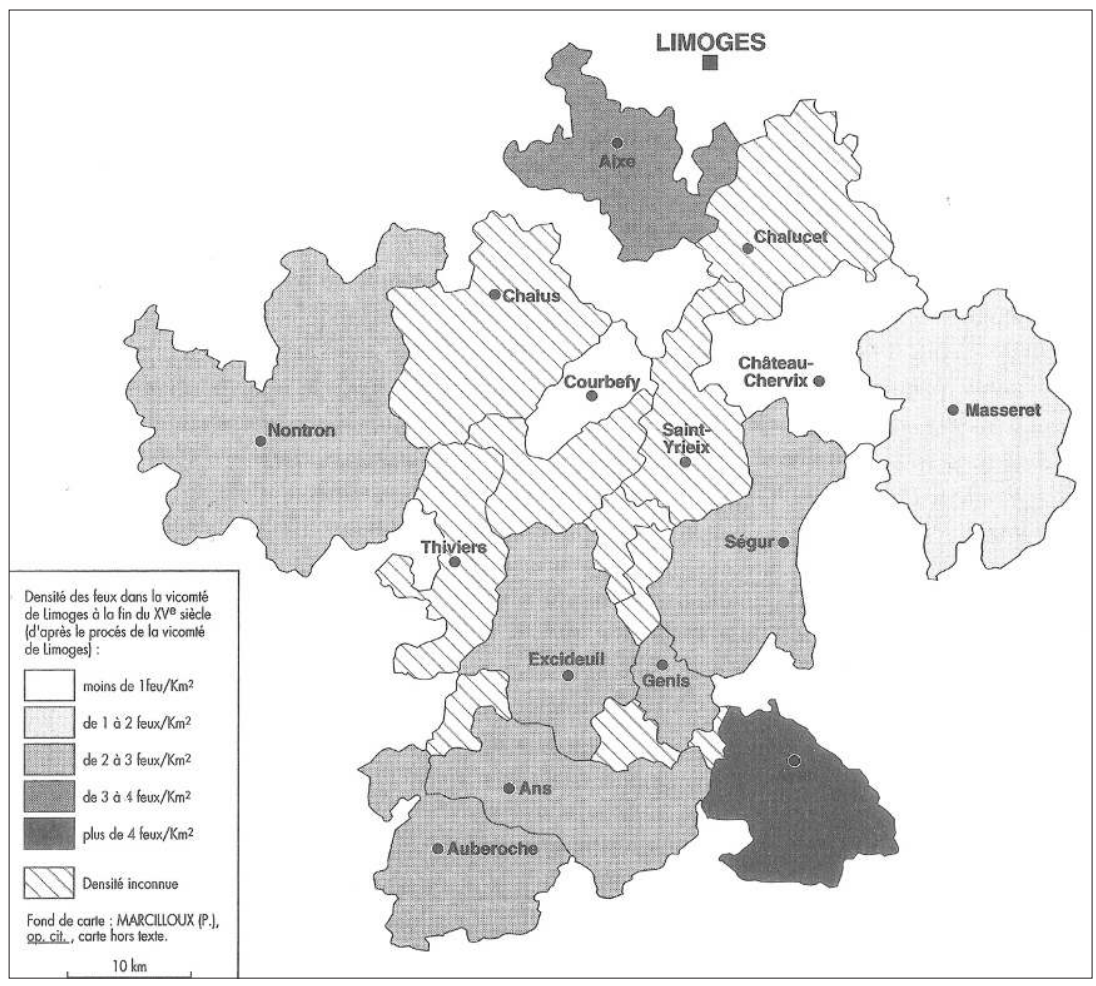

(d'après TRICARD, Jean, Les campagnes limousines..., op. cit., p. 242, carte 2)

viennent de dix d'entre elles entre 1344 et 1347. L'importante châtellenie d'Auberoche était aux mains des Anglo-Gascons dès l'été $1345^{26}$, sa perte étant actée par Charles de Blois, en janvier 1347, à la suite d'une transaction très compliquée impliquant Philippe VI et le cardinal Talleyrand de Périgord et ponctuée par sa vente pour la somme de 20000 florins (probablement utilisés pour réduire les dettes de Charles et Jeanne contractées envers le roi $)^{27}$. La carte de Roblin montre aussi dix autres châteaux vicomtaux secondaires, dont quelques-uns sont mentionnés dans nos comptes, mais aussi Mauscle (Génis-Moruscle) et Thiviers comme des seigneuries contribuant aux recettes vicomtales. Une petite poignée des seigneuries était tenue par d'autres familles seigneuriales, dont quelques-unes résidaient en dehors de la vicomté mais recevaient, de temps en temps, des dons des

26. GRIBIT, Nicholas A., Henry of Lancaster's Expedition to Aquitaine, 1345-46. Military service and professionalism in the Hundred Years War, Woodbridge, The Boydell Press, 2016, p. 120-125 et 130-132 pour la bataille et l'occupation d'Auberoche.

27. Jones, Michael, Recueil des actes de Charles de Blois..., op. cit., $\mathrm{n}^{\circ} 88$. 
vicomtes; celle de Lastours formait une enclave indépendante entre les châtellenies d'Aixe et de Chalus ${ }^{28}$.

L'enquête nationale, bien connue, de 1328 au sujet des feux sur lesquels des taxes pourraient être imposées, montre que le Limousin était l'une des régions du royaume la moins densément peuplée ${ }^{29}$ (fig. 2). Dans le HautLimousin, à l'ouest de la ville de Limoges, pourtant située dans la vallée fertile de la Vienne, la châtellenie d'Aixe n'avait que quelque $3,3 \mathrm{feux} / \mathrm{km}^{2}$, alors que son économie bénéficiait vraisemblablement de sa situation sur une rivière navigable et de sa position proche du centre urbain le plus important de la vicomté. Ailleurs, en Bas-Limousin, la châtellenie d'Ayen possédait plus de $6 \mathrm{feux} / \mathrm{km}^{2}$, ce qui était proche de la moyenne nationale. Mais d'autres châtellenies comme Auberoche, Ans, Excideuil et Ségur n'avaient que 2 à 3 feux $/ \mathrm{km}^{2}$, alors que Masseret ne comptait que 1,4 feu/ $\mathrm{km}^{2}$ et Château-Chervix moins d'un feu $/ \mathrm{km}^{2}$. Les villes étaient petites et les communications souvent difficiles. Dans le sud-ouest de la vicomté se trouvaient des sols plus riches et les contacts économiques avec la Guyenne étaient actifs, mais ailleurs, beaucoup d'autres parties de la vicomté ne disposaient que de sols pauvres, dont la plupart se situait au-dessus de 300 ou même 400 mètres d'altitude. La culture des céréales y était souvent problématique, la vigne absente, aussi l'élevage était l'activité agricole principale. En 1491, par exemple, on indiquait que Masseret était " la plus pauvre [châtellenie] de la Guyenne ", " un pays de forêts, où la paisson, seule, pourrait rapporter gros ${ }^{30} "$.

Quel est l'apport de nos fragments de comptes? Écrits sur des feuilles de papier cousues ensemble pour former des rôles, ceux de Pierre Molin ont survécu en deux copies contemporaines, l'une en français rédigée par un clerc de langue d'oïl, l'autre, par un clerc connaissant la langue d'oc (fig. 3) Mais il n'est pas facile de situer l'une par rapport à l'autre ces deux versions du même compte $\left(\mathrm{A}^{1} \text { et } \mathrm{A}^{2}\right)^{31}$. Si l'une $\left(\mathrm{A}^{1}\right)$ semble être, sans certitude aucune, un brouillon, l'autre $\left(\mathrm{A}^{2}\right)$, malgré son apparence formelle plus soignée, n'en est pas l'exacte mise au net : elle comporte aussi, comme $\mathrm{A}^{1}$, des ratures et n'en reprend pas tous les articles (cinquante-sept pour des

28. Gouffier de Lastours était l'un des nobles qui ont agi comme garants pour un prêt fait à Charles par la compagnie des Malabayala en 1345 (JonEs, Michael, Recueil des actes de Charles de Blois..., op. cit., $\left.\mathrm{n}^{\text {os }} 66,68,69\right)$. Au temps de l'administration de Jeanne et Charles, Chalus (avec Courbefy) ne faisait plus partie du domaine des vicomtes (CLÉMENTSimon, Gustave, La vicomté de Limoges, géographie et statistique féodales, Périgueux, Cassard Frères, 1873, p. 108-109).

29. La vicomté comporte 234 paroisses et 25421 feux en 1328 (LOT, Ferdinand, « L'État des paroisses et des feux de 1328 ", Bibliothèque de l'École des chartes, t. xc, 1929, p. 54, voir aussi p. 89-91, 305-308). Pour la distribution et la perte de feux jusqu'à 1500, voir TRICARD, Jean, Les campagnes limousines..., op. cit., p. 88-96 et p. 241-243, annexe IV.

30. Ibidem, p. 189-190.

31. $\mathrm{A}^{1}=$ Arch. dép. des Pyrénées-Atlantiques, E $624 \mathrm{n}^{\circ}$ 2, un rouleau de cinq feuilles, dont la première est légèrement abîmée et dont il manque au moins une feuille après la cinquième; $\mathrm{A}^{2}$ = Arch. dép. des Pyrénées-Atlantiques, E $624 \mathrm{n}^{\circ}$ 1, un rouleau de dix feuilles (onze à l'origine). 
Figure 3 - Extrait des comptes de Pierre Molin le jeune, receveur dans la vicomté de Limoges

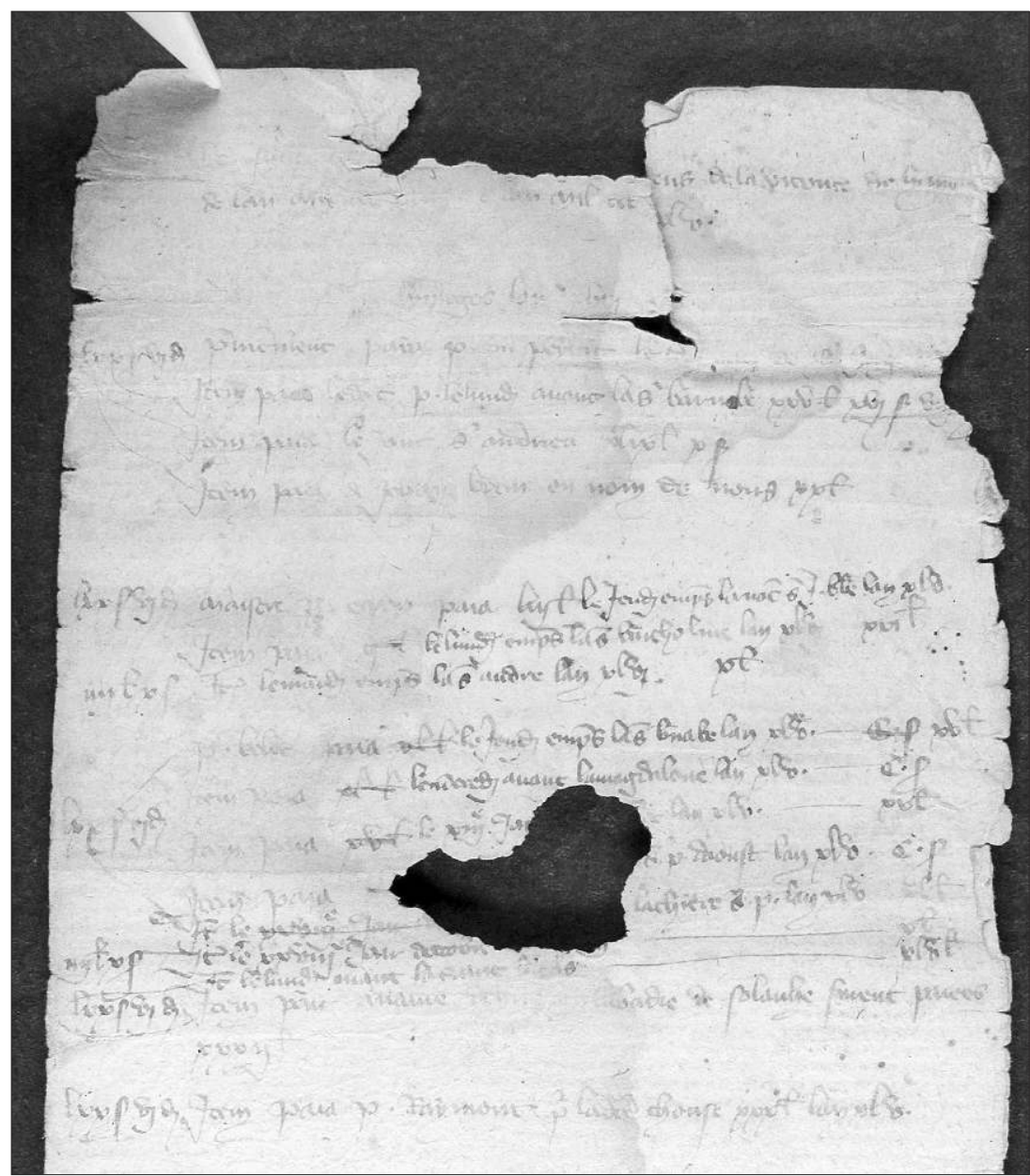

(Arch. dép. des Pyrénées-Atlantiques, E 624, no 2, art. 1-5)

recettes, et soixante-six pour des dépenses dans l'édition récente) (fig. 4). Elle a, malgré tout, été choisie pour base de l'édition, et on trouvera en note les articles non recopiés ${ }^{32}$. Les variantes orthographiques n'ont pas été, sauf exceptions notables, relevées. Deux sommes (" $70 \mathrm{~s} 6 \mathrm{~d}$ " et " $4 \mathrm{l}$ $10 \mathrm{~s}$ ") figurent, dans les deux versions, en marge de nombreux articles : elles portent tantôt sur un seul d'entre eux, tantôt sur plusieurs réunis dans ce cas par une accolade. Leur signification n'a pu être résolue. Afin de ne

32. Jones, Michael et Charon, Philippe, Comptes du duché de Bretagne..., op. cit., p. 403416. 
Figure 4 - Extrait des comptes de Pierre Molin le jeune, receveur dans la vicomté de Limoges

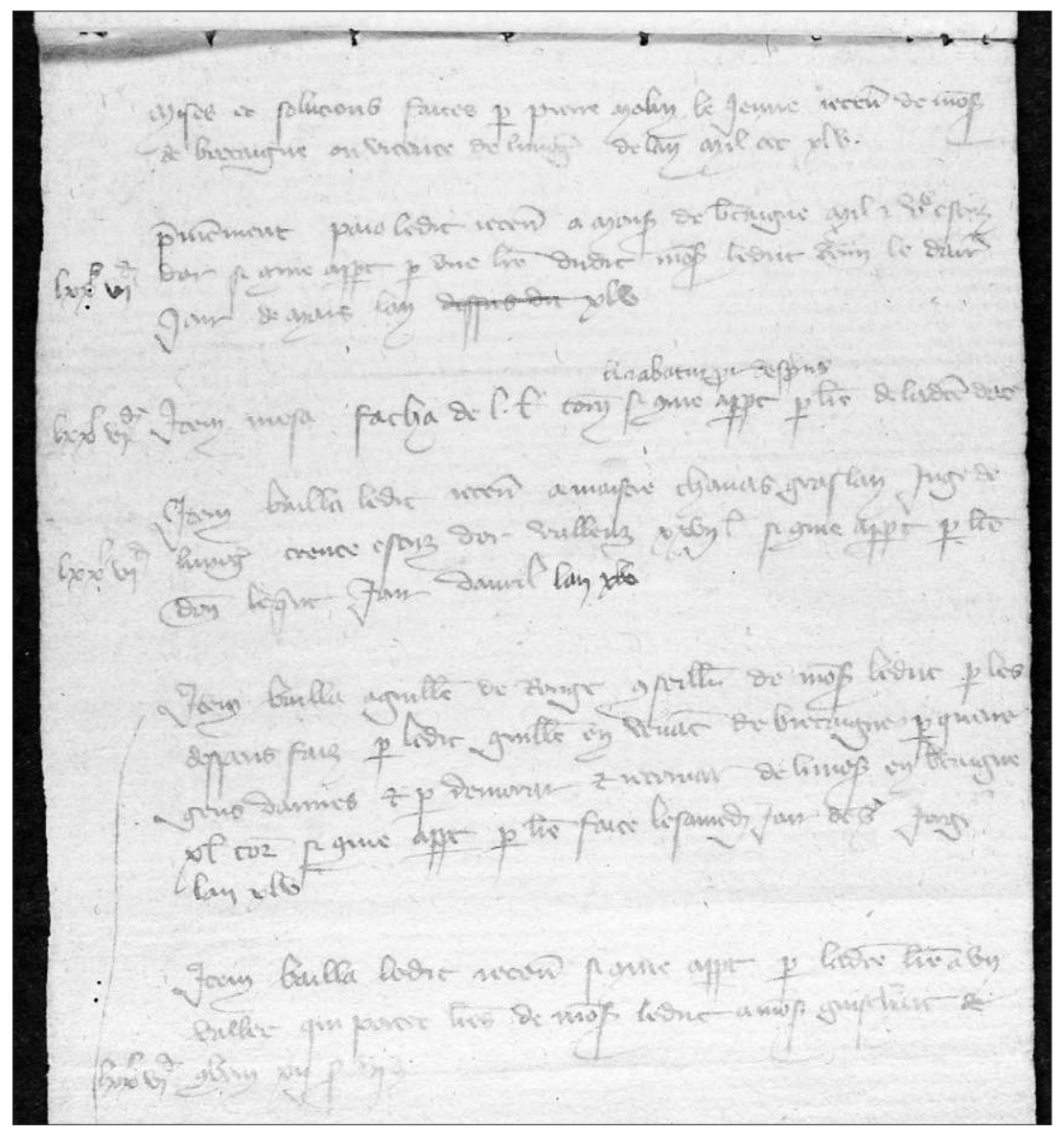

(Arch. dép. des Pyrénées-Atlantiques, E 624, no 1, art. 58-65)

pas surcharger l'édition, ces sommes n'y figurent pas. De même, n'ont pas été signalées les réunions d'articles par d'autres accolades ni éditées les petites marques en face d'articles, tels que des croix ou des cercles, choix arbitraires mais qui correspondent à l'esprit de l'édition qui privilégie le fond sur la forme. Si une autre option avait été prise, il aurait fallu aussi indiquer les passages qui semblent avoir été ajoutés après coup, du moins si le changement de couleur d'encre autorise une telle interprétation. La section concernant les dépenses (" mises ", à partir de l'article 58) ne figure que dans $\mathrm{A}^{2}$, mais elle devait aussi être présente dans $\mathrm{A}^{1}$ car la dernière feuille conservée porte, au bas, des marques de piqûres. Comme déjà mentionné, les comptes de Molin donnent des détails sur treize recettes, dont dix sont, 
suivant Roblin, des centres importants d'administration; par contre, deux châtellenies ne figurent pas sur sa carte, Mauscle (maintenant Génis) et Thiviers. Étant donné l'état fragmentaire de deux rôles, $\mathrm{A}^{1}$ et $\mathrm{A}^{2}$, il est possible que l'on ait reçu des revenus des châtellenies manquantes mais les feuilles ont été perdues; simplement, nous ne les connaissons pas.

Pour les châtellenies principales, des paiements furent faits habituellement au receveur, Molin, par plusieurs comptables différents, entre lesquels des prévôts locaux, ou d'autres pour eux, bien que le statut de la plupart des comptables ne soit pas indiqué, sauf pour des "bailles de taillee " d'Aixe et les " bailles " de Château-Chervix et de Frayssenet. Ainsi, à Limoges et à Aixe où les recettes pour les années 1344 et 1345 sont identifiées, à Limoges en 1344, quatre comptables sont nommés, et en 1345 cinq, dont un seul, Maître Pierre Gaudin, a compté dans l'année précédente. À Aixe, en 1344, il y a cinq comptables, et en 1345, neuf, dont deux ont agi en 1344 (Jean Baunelh, Géraut Bornazeu). Pour les autres recettes, il y a une seule entrée, bien que l'on compte quelquefois pour deux années séparément. À La Roche-l'Abeille, par exemple, Guy de Mauhville a compté pour des recettes en 1345 et en 1346 et il a aussi fait des paiements pour d'autres comptables. À Masseret, quatre hommes ont fourni de l'argent en 1345-1346, à Ségur sept l'ont fait, y compris le prévôt Aymeri de Bonneval. Pour le reste des châtellenies, il y a un seul comptable, sauf pour Excideuil où ils furent deux et à Ayen où l'on trouva trois. Il est très frustrant que la source des revenus qu'ils ont payés ne soit jamais indiquée, seule la somme d'argent étant mentionnée. Les montants totaux, en livres tournois, sont rassemblés ci-contre ${ }^{33}$.

Ces chiffres nécessitent un traitement minutieux, et des ambiguïtés demeurent, comme le révèlent les chiffres entre crochets. Par exemple, plusieurs entrées dans $\mathrm{A}^{1}$ contiennent des éléments qui ont été rayés ou qui ne figurent pas dans $\mathrm{A}^{2}$. Les écarts les plus importants se produisent dans l'entrée pour La Roche-l'Abeille, où des recettes dépassant 180 livres ont d'abord été enregistrées puis barrées. À Aixe plus de 66 livres ont été déduites de cette façon, et 23 livres à Masseret. Était-ce parce que les recettes présumées, d'un montant de plus de 300 livres, n'avaient pas été livrées? ou bien, si elles l'avaient été, est-ce que les entrées ont ensuite été rejetées, mises sous une autre rubrique, ou traitées par un autre officier? Notre impression est qu'au moins une partie en avait effectivement été recueillie, bien qu'elle ne soit pas clairement prise en compte dans les fragments survivants. Il est également évident à partir des comptes de Ségur (fig. 6) que Molin a reçu seulement une petite part (moins de $20 \%$ ) de l'argent géré par le prévôt local, qui a enregistré des recettes totales de 629 livres 14 sous $7 \frac{1}{2}$ deniers, et des dépenses de 626 livres 16 sous

33. Une version de ce tableau et une partie de l'analyse qui suit ont paru dans GRAHAMGOERING, Erika, "Negotiating princely power in late medieval France : Jeanne de Penthièvre, duchess of Brittany (c.1325-1384) ", dactyl., thèse de doctorat en histoire, sous la direction de Craig TAYLOR, université de York, 2016, p. 87-90. 
Figure 5 - Tableau des sommes reçues des châtellenies de la vicomté de Limoges, 1344-1347

\begin{tabular}{|c|c|c|}
\hline Territoire & Somme & Dates \\
\hline Limoges & $\begin{array}{l}1241 \mathrm{l} .16 \mathrm{~s} .8 \mathrm{~d} .+ \\
89 \mathrm{l} .10 \mathrm{~s} .6 \mathrm{~d} \text {. faible monnaie } \\
\text { (ou } 1304 \mathrm{l} .16 \mathrm{~s} .8 \mathrm{~d} \text {. } \\
+67 \mathrm{l} .11 \mathrm{~s} .4 \mathrm{~d} \text {. faible monnaie) } \\
\text { En raison des incertitudes provoquées par } \\
\text { des dégâts au manuscrit, nous avons placé } \\
\text { entre parenthèses des sommes alternatives } \\
\text { possibles. }\end{array}$ & $\begin{array}{l}8 \text { juin } 1344- \\
7 \text { décembre } 1346\end{array}$ \\
\hline Aixe-sur-Vienne & 316 l. $14 \mathrm{~s}$. & $\begin{array}{l}22 \text { avril } 1345- \\
1^{\text {er }} \text { janvier } 1347\end{array}$ \\
\hline $\begin{array}{l}\text { La Roche- } \\
\text { l'Abeille }\end{array}$ & $\begin{array}{l}421 \mathrm{l} .12 \mathrm{~s} . \\
\text { (ou } 395 \mathrm{l} .14 \mathrm{~s} .+10 \text { l. petite monnaie) }\end{array}$ & $\begin{array}{l}12 \text { juillet } 1345- \\
19 \text { janvier } 1347\end{array}$ \\
\hline Château-Chervix & 123 l. 15 s. 3 d. & $\begin{array}{l}4 \text { septembre } \\
1346\end{array}$ \\
\hline Masseret & 3191. & $\begin{array}{l}27 \text { avril } 1345- \\
25 \text { novembre } \\
1346\end{array}$ \\
\hline $\begin{array}{l}\text { Saint-Yrieix } \\
\text { (-la-Perche) }\end{array}$ & $23 \mathrm{l}$. & s.d. \\
\hline $\begin{array}{l}\text { Ségur } \\
\text { (-le-Château) }\end{array}$ & 119 l. 0 s. 4 d. & 3 janvier 1346 \\
\hline Nontron & $37 \mathrm{l} .6 \mathrm{~s}$. & s.d. \\
\hline Thiviers & $10 \mathrm{l} .10 \mathrm{~s}$. & s.d. \\
\hline Excideuil & 1221. & s.d. \\
\hline $\begin{array}{l}\text { Mauscle } \\
\text { (Génis-Moruscle) }\end{array}$ & $21 \mathrm{l}$. & s.d. \\
\hline Ans & $701.5 \mathrm{~s}$. & s.d. \\
\hline Ayen & 2271. & $\begin{array}{l}23 \text { mai } 1345- \\
9 \text { juin } 1346\end{array}$ \\
\hline Total & $\begin{array}{l}3052 \text { l. } 19 \text { s. } 3 \text { d. + } \\
89 \text { l. } 10 \text { s. } 6 \text { d. faible monnaie } \\
\text { (ou } 3190 \text { l. } 1 \text { s. } 3 \text { d. + } \\
77 \text { l. } 11 \text { s. } 6 \text { d. faible monnaie et } 334 \text { l. } 3 \text { s. biffé) }\end{array}$ & $\begin{array}{l}8 \text { juin } 1344- \\
19 \text { janvier } 1347\end{array}$ \\
\hline
\end{tabular}

6 deniers en argent (ainsi que des recettes considérables en nature) ${ }^{34}$. Si tel était le cas général, les comptes de Molin ne donnent qu'une vue très partielle des revenus de la vicomté et de la valeur de châtellenies individuelles.

34. Annexe, art. 47-54, un total de 3815 1/4 septiers de blé, y compris de froment, d'avoine et de seigle. 


\section{Figure 6 - Extrait des comptes pour Ségur 1345-1346}

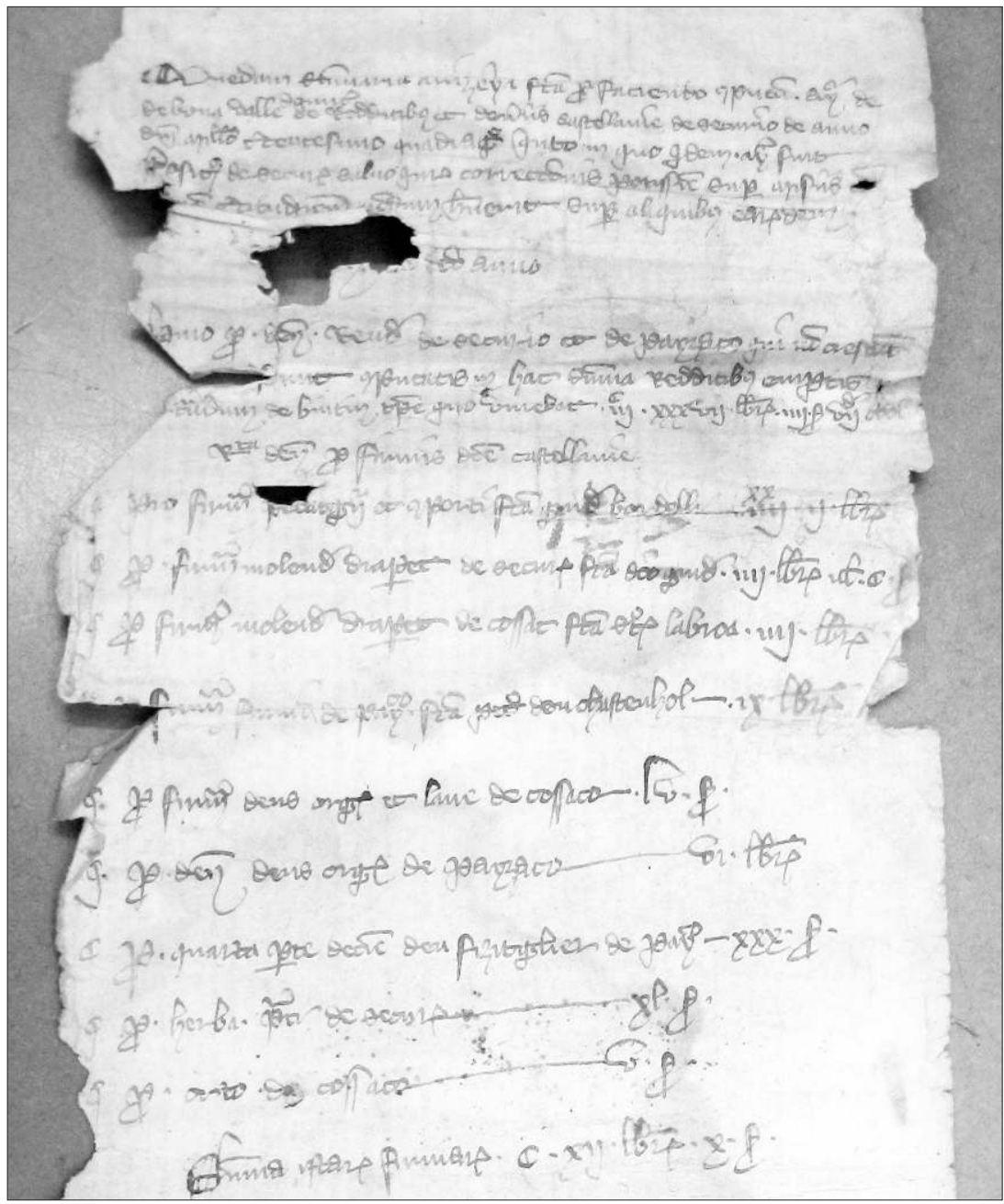

(Arch. dép. des Pyrénées-Atlantiques, E 863, édités ci-dessous, annexe, art. 1-5)

Cette conclusion est renforcée par le fait que les rendements enregistrés pour plusieurs châtellenies étaient sans commune mesure avec leur importance physique ${ }^{35}$.

35. Nous utilisons ici les paroisses comme une mesure de la taille des châtellenies plutôt que les feux $/ \mathrm{km}^{2}$ discutés ci-dessus, non seulement parce que cette information est plus complète, mais parce que la paroisse offre une mesure légèrement plus stable que la densité de population, ce qui aide à compenser la date tardive des données. Cependant, en supposant que la répartition des feux ait été à peu près proportionnelle à travers 
Ans, comprenant dix-sept paroisses, était parmi les plus importantes de la vicomté, mais elle occupe seulement le huitième rang en fonction des recettes reçues par Molin. Pourtant, Ans a rapporté près du double des revenus de Nontron, alors qu'elle ne représentait que la moitié de sa superficie. L'explication de ces discordances repose en partie sur la turbulence croissante que cette région connaissait pendant la période concernée par les comptes de Molin. Ces deux châtellenies d'Ans et de Nontron, par exemple, ont cédé aux forces anti françaises au cours de l'été 1345, la dernière par la trahison de son capitaine, Ytier de Magnac, qui a livré le château aux Anglais ${ }^{36}$. D'Excideuil, l'une des plus grandes châtellenies, est

\section{Figure 7 - Recettes des châtellenies dans les comptes de Pierre Molin, receveur dans la vicomté de Limoges 1344-1347, par rapport à leur taille en paroisses ${ }^{37}$}

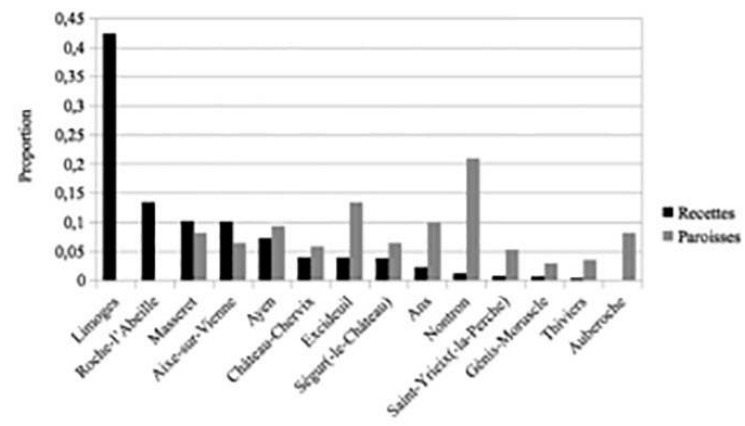

les châtellenies au XIV ${ }^{\mathrm{e}}$ siècle, on peut noter que cela ne remet pas en cause les écarts observés ici. Les châtellenies de Ségur, de Moruscle, d'Excideuil, d'Ans, d'Auberoche, et de Nontron, eurent toutes entre 2 et 3 feux $/ \mathrm{km}^{2}$, ce qui ne peut expliquer la variation entre leurs paiements à Molin. En revanche, les populations élevées d'Aixe et d'Ayen auraient pu contribuer à leurs recettes relativement élevées d'une manière non suggérée par leur nombre de paroisses; mais Château-Chervix, dont la population était la moins dense de toutes ces châtellenies, les suivait en revenus. Il reste donc à identifier d'autres explications pour ces déséquilibres.

36. Sumption, Jonathan, The Hundred Years War I : Trial by Battle, Londres, Faber and Faber, 1991, p. 457-458 pour la prise d'Ans et Nontron par les Anglo-Gascons. La " guerre de Nontron " figure dans les comptes d'Ayméri de Bonneval (ci-dessous, annexe, art. 14, 20, 57, 63, 64).

37. Pour le nombre des paroisses, voir ClÉMENT-SIMON, Gustave, La vicomté de Limoges..., $o p$. cit. Il ne donne aucun dénombrement de La Roche-l'Abeille, qu'il considère comme faisant partie de la châtellenie d'Aixe; ce dernier a donc une représentation proportionellement gonflée dans notre graphique. D'autre part, il est inutile de comparer à l'aide de ces chiffres la châtellenie urbaine de Limoges avec le reste de la vicomté. 
revenue une somme dérisoire qui a été en grande partie annulée par les frais de sa défense ${ }^{38}$. Auberoche, avant même sa capture par les Anglais, n'a en rien contribué aux recettes de 1344 à 1345 . En conséquence, nos chiffres ne doivent pas être considérés comme définitivement représentatifs des revenus de la vicomté. Ils fournissent simplement des indications générales sur les ressources disponibles pour le duc et la duchesse dans leur vicomté pendant les premières années de leur gouvernement, et sur la manière dont ils les ont utilisées.

En ce qui concerne les dépenses qu'a effectuées Molin, ses comptes portent sur une période plus courte que celle des revenus enregistrés (environ dix-huit mois au lieu de trente-et-un). Du 31 mars 1345 au 23 septembre 1346, Molin a déboursé environ 3919 livres tournois. La première des "mises " qu'il a enregistrées constitue le paiement le plus élevé, d'un montant de 1500 écus (ou 1350 livres tournois) livrés directement à Charles de Blois ${ }^{39}$. Molin lui a également versé 800 écus (ou 720 livres tournois) au nom des habitants de Limoges à la fin mai 1345 - s'agissait-il d'un prêt ou d'une amende? - et le duc a reçu 240 écus (196 livres tournois) de plus en juin ${ }^{40}$. Outre ces exemples, il n'y a que six autres dépenses s'élevant entre 100 et 200 livres tournois qui ont été enregistrées sur un total de quatre-vingt-huit paiements ici détaillés. La plupart de ces sommes couvraient les frais administratifs au sein de la vicomté. Olivier du Guesclin ${ }^{41}$, Guillaume de Rougé ${ }^{42}$ et Rainfroi Le Voyer, archidiacre de Rennes ${ }^{43}$, qui avaient été envoyés de Bretagne à Limoges pour y surveiller des réformes à la fois civiles et militaires, ont reçu 165 livres tournois

38. Jones, Michael et Charon, Philippe, Comptes du duché de Bretagne..., op. cit., p. 412413 art. 85-91 et ci-dessous, annexe, art. 14.

39. Ibidem, p. 410 art. 58 (31 mars 1345). Comme le démontre l'itinéraire ducal présenté par Jones, Michael, Recueil des actes de Charles de Blois..., op. cit., Charles, dans le cadre d'un voyage qui l'avait conduit à Avignon où le pape résidait à cette époque, était arrivé à Limoges le 9 mars; en avril, nous le retrouvons de nouveau en Bretagne.

40. Jones, Michael et Charon, Philippe, Comptes du duché de Bretagne..., op. cit., p. 412 art. 65,78 .

41. Olivier, seigneur de la Ville-Anne (Ille-et-Vilaine), chevalier, fut un frère puÏné de Robert du Guesclin, seigneur de Broons, le père du connétable, Bertrand du Guesclin (LucE, Siméon, Histoire de Bertrand du Guesclin..., op. cit., p. 21, note 3). Au début de la guerre de Succession il a suivi la partie de Jean de Montfort et fut envoyé en Angleterre où, avec Bernard de Guignan, il a négocié un accord avec Édouard III pour Jeanne de Flandre (Jones, Michael, "Some documents... ", art. cit., p. 72), mais il a reçu des lettres de rémission de Charles de Blois le 31 décembre 1344 "pour plusours exces et malefices durans les guerres et rebellions de Bretaigne " (JonEs, Michael, Recueil des actes de Charles de Blois..., op. cit., $\left.\mathrm{n}^{\circ} 48\right)$.

42. Receveur général de Bretagne en 1337 (BnF, ms fr. 26291, no 372) et toujours un conseiller important du duc en 1360 (JonEs, Michael, Recueil des actes de Charles de Blois..., op. cit., $\mathrm{n}^{\circ}$ 241).

43. Peut-être chancelier de Bretagne avant Gautier de Saint-Pern, comme chantre de Dol, il était toujours en vie comme membre d'une ambassade à Édouard III en 1359 (JonES, Michael, Recueil des actes de Charles de Blois..., op. cit., p. 33 et no 217). 
au début de $1346^{44}$. Le sénéchal et gouverneur, Guillaume de La Marche, a reçu 163 livres 7 sous 4 deniers en juillet $1346^{45}$ tandis qu'à peu près au même moment Jean de La Porte, capitaine de la vicomté, a obtenu 120 livres tournois (ou peut-être 140 livres), ainsi que d'autres sommes moins importantes ${ }^{46}$. Mais en sus des versements faits au duc, il n'y a que deux paiements importants effectués au-delà de la vicomté; ceux-ci sont faits à la demande de Jeanne de Penthièvre pour son propre compte, l'un de 106 écus d'or 15 sous 3 deniers en mars 1345, et l'autre de 76 livres 6 sous 1 denier tournois en août $1346^{47}$. En effet, au cours d'une période de hausse des coûts militaires et des difficultés économiques, la plupart des dépenses du receveur portaient sur le paiement des salaires au personnel, sur les dépenses militaires, sur l'expédition des messagers et sur de petits frais divers, y compris des aumônes et l'achat de chevaux.

Regardons ces dépenses d'un peu plus près. Parmi les agents de la vicomté qui recevaient des salaires réguliers figurent le juge de Limoges (maître Thomas Graslin), le procureur (maître Guillaume Texue), et le prévôt (mons. Foulquieux), tandis que maître Geoffroy de Champaigne, juge de Périgord, a également reçu 10 livres et 5 livres tournois " par ses gages " en $1346^{48}$. Douze autres "maîtres " sont mentionnés dans les comptes probablement tous des diplômés universitaires, dont plusieurs, semble-t-il, étaient également avocats ${ }^{49}$. Toujours dans le cadre de l'administration de la justice, des paiements à Johanot Le Gascon pour l'exécution des criminels, y compris Hugues d'Anse en 1345, peuvent être notés ${ }^{50}$. Parmi ceux qui n'appartenaient pas au personnel de la vicomté mais qui bénéficiaient des versements du receveur, le plus éminent était le chancelier de Bretagne, Gautier de Saint-Pern, qui a visité Limoges en mai 1345, avant de se rendre à Paris pour une mission diplomatique ${ }^{51}$.

Viennent ensuite les dépenses militaires : des arbalètes ont été achetées à plusieurs reprises (ce qui indique peut-être une fabrication locale), dont soixante-deux ont été envoyées à Nantes dans le courant de l'année 1345, et dix à Excideuil en décembre 1345 ou janvier $1346^{52}$. Là, quinze

44. Jones, Michael et Charon, Philippe, Comptes du duché de Bretagne..., op. cit., p. 413 art. 101; voir aussi art. 94, 96-98 et p. 414 art. 108 et ci-dessous, annexe, art. 22-24, 27, 59, 66 et 75 pour l'action et les dépenses des réformateurs en Limoges.

45. Ibid., p. 416 art. 128.

46. Ibid., p. 416 art. 42.

47. Ibid., p. 414 art. 110, " en espices ", et p. 416 art. 131, " en espieces ", que nous traduisons comme " espèces " plutôt que " épices".

48. Ibid., p. 404 art. 6 note, p. 410 art. 60, p. 414 art. 120, 121 et p. 415 art. 127 (Graslin); p. 414 art. 112, p. 415 art. 122, 416 art. 144 (Texue); p. 413 art. 94 (Foulquieux); p. 414 art. 113 et p. 415 art. 134 (Champaigné).

49. Ibid., p. 389 art. 2, p. 390 art. 6 , p. 391 art. 11, p. 393 art. 24 et 29, et note 89, p. 395 art. 43, p. 396 art. 57, p. 398 art. 74, p. 401 art. 124, p. 402 art. 141 et 142.

50. Ibid., p. 411 art. 67 et 71 .

51. Ibid., p. 411 art. 68, p. 413 art. 93, 94, 102. Il fut plus tard évêque de Vannes, 1347-1359.

52. Ibid., p. 411 art. 70 (Nantes) et p. 412 art. 85 (Excideuil). Pour d'autres dépenses militaires contractées à Excideuil en juin, juillet et août 1345, voir ci-dessous, annexe, art. 14, 19. 
arbalétriers les attendaient, tandis que douze autres ont été mis en la garnison de Ségur, probablement en mai $1346^{53}$. Les comptes de Bonneval pour cette châtellenie apportent des informations supplémentaires considérables sur la taille et le coût de la garnison, des armes, et des réparations à Ségur dans les mois précédant immédiatement leur arrivée. Ils mettent également en évidence le rôle d'Olivier du Guesclin et Guillaume de Rougé, "réformateurs " de Limoges, dans l'organisation des défenses, tandis que Molin réglait une partie de leurs dépenses " en visitant les chasteaux " en février $1346^{54}$. Des seigneurs locaux semblent avoir été conservés à des fins militaires, comme mons. Guischart de Comborn, seigneur de Treighnac, qui a reçu 50 livres tournois pour son salaire en septembre $1345^{55}$. Le mercenaire génois, Baldo Doria, était entré au service de Charles et Jeanne avant décembre 1345 et il resta avec eux pendant de nombreuses années avant de finir ses jours après 1369 dans celui de Charles $\mathrm{V}^{56}$. Il a reçu plusieurs paiements de Molin (d'un montant dépassant 200 livres tournois) bien qu'il n'ait probablement jamais visité la vicomté : il envoyait plutôt ses représentants pour récupérer son argent, puisque sa principale zone d'opération était dans le pays de Retz ${ }^{57}$.

Par contre, les seuls religieux ayant reçu un don de bienfaisance du duc comme vicomte au cours de la période concernée par les comptes furent les dominicains de Limoges, et ceci de 5 livres tournois seulement. ${ }^{58} \mathrm{Cela}$ peut étonner étant donné le zèle religieux si connu de Charles de Blois et le mécénat qu'il pratiquait en Bretagne ${ }^{59}$. Mais il n'y avait peut-être pas toujours les ressources pour répondre à des besoins non essentiels dans la vicomté. En ce qui concerne les dépenses, nous pouvons constater à partir des fragments subsistants que le receveur versait presque le double de ce qu'il avait reçu. Selon des moyennes certes un peu grossières, la vicomté apportait à Charles et Jeanne des sommes légèrement inférieures à 100 livres tournois par mois, alors qu'ils dépensaient environ 220 livres tournois. Toutefois, comme les comptes de Ségur le démontrent, les chiffres de Molin ne représentent pas l'ensemble des finances vicomtales.

53. Ci-dessous, annexe, art. 29, 31, 40.

54. Jones, Michael et Charon, Philippe, Comptes du duché de Bretagne..., op. cit., p. 413 art. 97.

55. Ibid., p. 412 art. 75. En 1345 il fut donné par le duc et la duchesse « la haute et basse justice des lieux, villes et terres qu'il possède dans leur vicomté de Limoges " (JonEs, Michael, Recueil des actes de Charles de Blois..., op. cit., $\left.\mathrm{n}^{\mathrm{o}} 76\right)$.

56 . BnF, pièces originales 1015 , dossier 23184, $\mathrm{n}^{\circ} 3,31$ août 1369 , quittance de Baude Doria (Dore), chevalier, patron de la " galye "Sainte-Katherine, de trois cordes, donnée à Richart de Brumare, garde des clos des galées à Rouen.

57. Jones, Michael et Charon, Philippe, Comptes du duché de Bretagne..., op. cit., p. 412 art. 82, 83, p. 413 art. 105, p. 414 art. 107, 109; il est bien présent dans les comptes de la châtellenie de Touffou, 1348-1352 (Ibid., p. 435-436, art. 78-90, 92-102, p. 437 art. 107-120).

58. Ibid., p. $412 \mathrm{n}^{\circ} 80$.

59. Voir les énumérations des dons rapportés par les témoins en vue du procès de canonisation de Charles en 1371 (SÉRENT, Antoine de, Monuments du procès de canonisation du bienheureux Charles de Blois, duc de Bretagne, 1320-1364, Saint-Brieuc, R. Prud'homme, 1921). 
Bien d'autres questions pourraient être soulevées : le monnayage et les taux de change ${ }^{60}$, les pratiques agricoles, le langage des comptes, la prosopographie des personnes nommées dans les comptes, l'administration du domaine vicomtal au cours des années suivantes ${ }^{61}$, pour n'en citer que quelques-unes. Mais nous espérons que nous en avons dit assez pour montrer que, bien que fragmentaires et isolés, ces rares survivants de dossiers financiers autrefois courants apportent une précision utile à notre compréhension des relations britanno-limousines dans une période critique au début de la guerre de Cent Ans.

60. Graham-Goering, Erika, Negotiating princely power..., op. cit., p. 88, note 14. Pour les pièces émises à Limoges au nom de Charles de Blois, voir JÉzÉQUEL, Yannick, Les monnaies des comtes et ducs de Bretagne du X $X^{e}$ au Xve siècle, Paris, Maison Florange, 1998, p. 257-259.

61. Quelques terres vicomtales furent vendues, comme Auberoche en 1347 (ci-dessus note 24), La Roche-l'Abeille en 1357 et Salon près de Masseret avant 1364 (JonEs, Michael, Recueil des actes de Charles de Blois..., op. cit., $\mathrm{n}^{\circ}$ 197, 295). 


\section{Annexe - Résumé préparé en vue de la présentation des comptes d'Ayméri de Bonneval, écuyer, prévôt de la châtellenie de Ségur-le-Château, 1345-1346}

Arch. dép. des Pyrénées-Atlantiques, E 863, papier, un rouleau de cinq feuilles, 2,70 m de long, endommagé au début.

\section{Feuille 1}

Quedam summaria avizeya facta pro faciendo computatis Aymericus ${ }^{62} \mathrm{de}^{63}$ Bona Valle, domicellus ${ }^{64}$, de redditibus et denariis castellanie de Securio ${ }^{65}$ de anno domini millesimo trecentisimo quadragesimo quinto in que idem Aymericus fuit prepositus de Securio salvo juris consuetudinis petiss' de super misiis ... certitudinem nondum habuerit super aliquibus earumdem.

\section{... dicto anno 66}

... Hamo pro denariis rendis de Securio et de Payzson ${ }^{67}$ qui non a est ....unt computatis in hac summa redditibus emptis ... [Ber]trandum de Bintin ${ }^{68}$ tempore quo vivebat 337 l. 4 s. $7^{1 / 2} \mathrm{~d}$.

\section{Recepta denarii per firmis dicti castellanie}

2 Pro firmis pedatgii et compoti facta Peraudi Bordelli

$82 \mathrm{l}$.

3 Pro firmis molendini draperet de Securio facta dicto Guidone

$4 \quad$ Pro firmis molendini draperet de Cossac facta Stephani Labroa $4 \mathrm{l}$.

5 [Pro] firmis furnis de Payzaco facta Petro dou Chasteuhol $9 \mathrm{l}$.

$6 \quad$ Pro firmis deus orger' et lane de Cossac $55 \mathrm{~s}$.

7 Pro denariis deus orger' de Payzsaco 61 .

$8 \quad$ Pro quarta parte decime deu fizitglier (?) de Payzaco $30 \mathrm{~s}$.

62. Abrégé comme $A y^{\prime}$ au long du document, la forme complète Aymericus se trouve vers la fin.

63. de répétée au début de la ligne suivante

64. domicellus interligné

65. Ségur-le-Château, département Corrèze, arrondissement Brive-la-Gaillarde, canton Lubersac.

66. La marge gauche est arrachée à cet endroit, et il y a aussi un petit trou dans le manuscrit.

67. L'orthographe préférée dans ce manuscrit est Payzsac : maintenant Payzac, département Dordogne, arrondissement Nontron, canton Isle-Loue-Auvézère, une petite commune d'un peu plus de 1000 habitants, située à environ 12 kilomètres au sud-ouest de Ségur par la route.

68. La famille de Bintin est originaire de la paroisse de Cintré (Ille-et-Vilaine) [www.infobretagne.com/cintre.htm]. Le 22 octobre 1306 Bertrand de Bintin, « fils de mons. Bertrand de Bintin, donna une quittance pour $10 \mathrm{l}$. feible monnaie " aux exécuteurs du testament du duc Jean II " pour le retour des fermes de Hede et pour toutes mes autres demandes " (Arch. dép. de Loire-Atlantique, E 21, nº 26). Est-ce que c'est lui qui est mentionné dans ce compte en 1345 ? 


\section{Summa istarum firmariorum}

$$
112 \text { l. } 10 \mathrm{~s} \text {. }
$$

11 Item lo fayun drech de castellanie de dicto anno fuit affirmatus eidem Aymericus pro maiori summa quam valuerent cum assizie dicte castellanie non fuerunt vente ut fieri debebat metu guerre vicecomitis Lemovicensis et inimicorum patrie quod fayun drech dicti anni potant valere in summa

12 Item recepit idem Aymericus a certis hominibus tailliabilibus dicte castellanie pro tallia eis inpositis et per manum prepositi levare consuetis 60 l. quod baiuli castellanie de tallia per eos levatis alibi et non dicto Aymerico responderunt prout in fine huius computi declarator.

Summa universalis tocius recepte denarii predictorum 629 l. $14 \mathrm{~s} .7 \mathrm{l} / 2 \mathrm{~d}$.

Sequntur soluciones et misie denarii facte per dictum Aymericum de premissis

13 Primo, 17 l. 16 s. 4 d. debitur Aymericum certis personis super redditibus dicte castellanie

[17 l. 16 s. 4 d.]

14 Item de mandato dominorum Gauffridi Helie et Guidi de Nova Villa, militibus, capitaneorum vicecomitis Lemovicensis, tradidit idem Aymericus pro eundo ad guerram de Nontronio ${ }^{69}$, videlicet apud Exidol ${ }^{70}$ pro expensis factis ibidem pro gentes armorum et servientes die jovis in maiorum festo beati Marcialis et diebus martii et mercurii sequentibus anno XL quinto ${ }^{71}$

19 l. 2 s. 9 d.

\section{Feuille 2}

15 Item pro facto dicte guerre de mandato domini Johannis La Porta, militis, capitan[ei] superioris vicecomitis Lemovicensis predicti idem Aymericus tradidit Helie Coteti

16 Item quando dictus dominus de Porta et comitiva suavisitaverunt castrum de Securio et ibi ordinavit stabilitam expendit $35 \mathrm{~s} .1 \mathrm{~d}$.

17 Item pro facto dicti guerre tradidit idem Aymericus eidem capitaneo apud Riberuni ${ }^{72} 6 \mathrm{~s} .8 \mathrm{~d}$. et in quatuor animalibus grossis apud Securium per Dulphie 4 l. 3 s. et in viginti quatuor arietibus

69. Nontron, département Dordogne, prise par une bande d'Anglo-Gascons en juillet 1345 .

70. Excideuil, département Dordogne.

71. La fête principale de saint Martial est le 30 juin, un jeudi de 1345, donc les dépenses ont été réalisées le 30 juin et les 5 et 6 juillet 1345 .

72. ? La Ribière, département Corrèze, environ 6 kilomètres au nord-ouest de Ségur. 
18 Item habuit idem dominus de Porta a dicti Aymerici per manum Johannis de Perduris de denariis rendualibus baylie de Brossaco

19 Item a die martii ante festum beate Marie Magdalene usque ad festum beati Medii de Augusto ${ }^{73}$ fuit idem Aymericus pro facto dicte guerre tam apud Exidol' et Tiverum ${ }^{74}$ quam apud Sanctum Perdulphum ${ }^{75}$ durante seigio cum septem hominibus armorum et eorum famularum et cum viginti servientes et ad certis munitionis (?) et animalibus que defferebant certa victualia et expendit idem Aymericus pro expensis factis per dictis gentibus quam pro certis necessariis ad opus hospicii dicti domini de Porta apud Riberuni quod eciam pro expensis sex viginti servientibus factis pro duos dies eundo et redeundo apud Exidol' usque ad Suneriam sexaginta et decem libras vel certa de quibus nondum plene computavit cum domino de Porta.

20 Item tradidit, de mandato dicti domini de Porta, Guillelmo La Marcha de Cossaco pro uno roncino quem idem Guillelmum amisit pro guerra de Nontronio

$10 \mathrm{l}$.

21 Item, de mandato dicti domini de Porta, tradidit idem Aymericus domino Guillelmo Coteti et Adem' Helie, capitaneis de Securio, pro custodia castri de Securi

$130 \mathrm{l}$.

22 Item tradidit idem Aymericus, de mandato domini Oliverii de Cloayquin et Guillemi de Rogeyo, refformatorum vicecomitis Lemovicensis, eisdem capitaneis de Securio $100 \mathrm{l}$.

23 Item ante Purificacionis beate Marie, ${ }^{76}$ de mandato dictorum refformatorum, tradidit viginti sex hominibus armorum custodientibus castri de Securio

$26 \mathrm{l}$.

24 Item tradidit, de mandato dictorum refformatorum, eisdem hominibus armorum a festo Purificationis beate Marie anno quadragesimo quinto usque ad sequens Pascha ${ }^{77}$

$100 \mathrm{l}$.

25 Item tradidit idem Aymericus, per mandatum Johannis de Quadris, Helie de Nova Villa, primo capitaneo de Securio, in vino 6 l. pro victualibus custodiencium dictum castrum

26 Item tradidit idem Aymerus apud Securium, per Dulphum, dominis Gauffrido Helie et Guido de Nova Villa, militibus, capitaneis vicecomitis predicta, predictis suis $43 \mathrm{~s} .4 \mathrm{~d}$.

27 Item tradidit idem Aymericus pro expensis factis apud Securium per quatuor dies per dominos Oliverium de Cloyquin et Guillelmum de Rogeyo et eorum comitivam 63 s. 8 d.

73. 21 juillet-15 août 1345 .

74. Thiviers, département Dordogne.

75. Saint-Pardoux-la-Rivière, département Dordogne, environ 8 kilomètres à l'est de Nontron.

76. 2 février 1346.

77. 2 février-16 avril 1346. 
28 Item expendit dominus de Castro Bocheti, senescallus vicecomitis Lemovicensis, apud Securium die veneris post festum sancti Marchi ${ }^{78}$

29 Item tunc de mandato domini senescalli venere in dicto castro duodecim balestarii de Lemovicensis cum filio Rollandi de Cusse qui ibi fuerunt per decem dies et expendit

$61.9 \mathrm{~s}$.

30 Item expendit idem dominus senescallus apud Securium cum comitiva sua computato quinto die Julii

31 Item de mandato Radulphi de la Thoscha habuerunt predicti balestarii a dicto Aymerico 39 s. circa festum beati Barnabe ${ }^{79} \quad$ [39 s.]

32 Sequntur expensis pro molendini, viz. pro tribus molis de Jauzaco et quadrigis eisdem, 60 s., et pro fargatcio

$30 \mathrm{~s}$.

33 Item pro faciendo de novo molendinum nomine draperet $^{80}$ de Securio $70 \mathrm{~s}$., et pro expensis quadriga $10 \mathrm{~s}$. ultimo quandem alia operas

34 Item pro quad' mola segalars et quadrig' $25 \mathrm{~s}$., et pro fargatgio dicti molendini

35 Item pro molendinum de Cossaco tam pro duabus molis segalars et quadrig' quam pro reparacione facta in molendino de Cossaco

37 Item expendunt quindecim latrones et proditorum sive epias ${ }^{82}$ capti apud Securio et tenti in carcere per diversa tempora $100 \mathrm{~s}$.

38 Item habuit Radulphus de la Tocha per manum Adem' Michael, baiuli, 24 l. de denariis rendualibus.

\section{Summa totalis solucionis et omnium misiarum predictarum} 626 l. $16 \mathrm{~s} .6 \mathrm{~d}$.

39 Item de mandato dominorum Gauffridi Helie et Guillelmi Adem', militibus, qui duxerunt et augent extranerum custodiam castri de Securio de decem servientes de Lemovicense, familiaribus domini Guichardi de Combornio, qui fuerunt in dicto castro per decem dies, habuerunt a dicto Aymerico

$7 \mathrm{l} .4 \mathrm{~d}$.

40 Item, de mandato capitaneorum de Securio et pro curia habuit, Philipus Auzelli pro se et undecim balestariis de Lemovicense pro duobus diebus apud Securio 24 s. $6 \mathrm{~d}$.

78. 28 avril 1346 .

79. 11 juin 1346.

80. En occitan : draper $=$ moulin à foulon.

81. Saint-Cyr-les-Champagnes, département Dordogne, arrondissement Nontron, canton Isle-Loue-Auvézère, située à environ 8 kilomètres au sud de Ségur.

82. En occitan : espia $=$ espions. 
41 Item ad tuicionem castri de Securio fecit fieri idem Aymericus in dicto castro certos chalassalles et certas reparacionem tam in portas et muris quam in fossatis et clausuris dicti castri et in eschura molendini et in aliis operibus que decostaverunt $30 \mathrm{l}$. alia compta

42 Item fecit fieri in dicto castro certos quayrelles et turnos balistariis et cordam putei que decostaverunt

$54 \mathrm{~s}$.

\section{Feuille 3}

Item sunt deducende eidem preposito summe que sequntur omni de eisdem computavit in sua compta

43 Primo, 40 s. pro herba parci de Securio cum gentes armorum existen' in castro de Securio devasteverunt dictam herbam omni animalibus suis

44 Item 4 l. de firma molendini draperet de Cosaco quod nichil operatus fuit in dicti anni cum chaussata molendini rupti foret

45 Item $50 \mathrm{~s}$. de firma molendini draperet de Securio in nichil fuit operatus a tempore quo Anglici rapuaverunt locum de Securio et telas dicti molendini.

[50 s.]

46 Item petit ut se deducantare 27 l. 2 s. 6 d. emptis per Bertrandum de Bintin tempore quo vivebat de quibus superius computavit in recepta denarii quos non potest habere et quas debebent, viz. ... La Marcha 20 l. quas idem Bertrandus petit se deduci pro vadiis suis et filio suo debitur pro custodia castri de Securio, et Guido Bordelli $100 \mathrm{~s}$. quas non potest solveret cum capitanei de Securio destruit fecerunt et hospicii pro meliorum tuicione dicti castri, et Guido de Mazeuris 42 s. 6 d. qui erat non solvendo et decessit.

[27 1. 2 s. 6 d.]

Summa istius deductionis quam petit fieri 35 l. 12 s. $6 \mathrm{~d}$.

\section{Feuille 4}

Recepta bladorum dicte castellanie de dicto anno XLV

47 De bladis rendualibus dicte castellanie que non crescent ne decrescunt 60 sext $^{\prime}{ }^{\prime 3}$ frumenti, 1721/4 sex' siliginis, 269 sext' avene.

48 Item pro firma molendini de Securio affirmata Guidoni Bordelli de Securio 50 sext' frumenti, 120 sext' modurae ${ }^{84}$.

49 Item pro firma molendini Sancti Cirici affirmata Guidoni Raffart 50 sex' frumenti, 100 sex' modurae.

\section{Summa modurarum}

$$
340 \text { sext' }
$$

83. Suivi de ce qui semble être viij

84. modura est normalement interprété comme une mesure (modius) mais ici et en dessous il indique clairement une sorte de culture de céréales, peut-être un mélange d'avoine et d'orge (comme le "dredge " anglais utilisé pour le brassage)? 
50 Item pro firma decimarum de Cossato affirmata Petro de Brolio et Guidonis Nepotis, 700 sex', viz. 34 sex' frumenti, 303 sex' siliginis, 303 sex' avene.

51 Item pro firma decimarum de Payzaco affirmata Petro Fulcherii, 1800 sex', viz. 200 sex' frumenti, 800 sex' siliginis, 800 sex' avene.

52 Item pro firma estivorum de Beyschenaco, ${ }^{85}$ Securio, Curia et Maxi facto magistro Petro Puihol, 170 sex' siliginis, 170 sex' avene.

53 Item pro decimarum terre Mauhini de Beyschenaco, 3 eminas siliginis.

54 Item pro firma decimarum Glandonio ${ }^{86}$ subastate supra Stephanum de Corbolus' (?) affirmata Petro Coteti deu Biart, 72 sex' siliginis, 37 sex' avene.

Summa tocius recepte omni bladorum predictorum et tocius annate predicte 3815 1/4 sex' bladorum, viz. 374 sex', 1 emina frumenti, 1521 1/4 sex' 1 emina siliginis, 1579 sex' avene, 340 sex' modurarum

Sequntur soluciones et misie facte per dictum Aymericum de bladis supradictis

55 Primo, 19 sex', 1 emina frumenti, 110 sex' silginis, 23 sex', 1 emina mod' 40 sex' avene debitur annuatim certis personis supra bayliam.

56 Item debent deduci pro minori mensura censual' de Cossac 4 sex' siliginis.

57 Item die veneris in festo Magdalene et sabbato sequenti ${ }^{87}$ desservi fecit idem Aymericus apud Riberumi 21 sex' frumenti in pane que fuit traditus dominis Johannis de Porta, Gauffrido Helie et Guidoni de Nova Villa, militibus, et quibusdam aliis ibidem pro facto guerre de Nontronio gagatis (?).

58 Item habuit dominus de Porta pro expensis factis per eum apud Securio cum comitiva sua 5 sex' avene minus quarta.

59 Item expenderunt cum equis apud Securio dominus Oliverius de Cloyquin et Guillelmus de Rogeyo cum comitiva sua 18 sex', 3 eminas avene.

$60^{88}$ Item habuit ${ }^{89}$ dominus de Castro Bocheti per manum Petri Fulcherii, clerici, de Payzaco 8 sex' frumenti circa nativitate beati Johannis le baptiste anno XL sexto ${ }^{90}$. Johanne de Boybic recipisse.

85. Beyssenac, département Corrèze, arrondissement Brive-la-Gaillarde, canton Lubersac, environ 4 kilomètres au sud de Ségur.

86. Glandon, département Haute-Vienne, environ 10 kilomètres au nord-ouest de Ségur. 87.22 et 23 juillet 1345 .

88. Immédiatement avant cet article, dans la marge de gauche, est écrit $b+$ Item indiquant l'omission à cet endroit de l'article 63 ci-dessous, qui est précédé dans la marge par $a+$.

89. Écrit au-dessus d'expendit, biffé.

90. Vers le 24 juin 1346. 
61 Item de mandato domini de Porta circa festum beati Medii ${ }^{91}$ habuerunt Gauffridus de Nova Villa et Ber' de Perussa, tunc capitanei de Securio, per mandato magistri Petri Puihol, 60 sex' siliginis et 60 sex' avene pro victualibus custod' dictum castrum.

62 Item et idem Ber' de Perussa habuit per mandato Guidonis Bordelli 4 sex', 1 emina frumenti.

63 Item expendit dominus de Castro Bocheti cum equis suis apud Securio in duabus vicibus 17 sex', 1 emina avene. Item eidem sappll' (?) et eidem comitiva 8 sex' avene quando de Securio versus Nontronum.

\section{Feuille 5}

64 Item, de mandato dicti domini de Porta, tradidit idem Aymericus Helie Coteti deu Biartz existenti in guerra de Nontronio 80 sex' siliginis, 40 sex' avene.

65 Item, de mandato dicti domini de Porta, a festo nativitate beate Marie usque ad sequens festo beate Lucie anno XL quinto, ${ }^{92}$ idem Aymericus tradidit pro provisione castri de Securio dominis Adem' Heli et Guillelmus Coteti, capitaneis de Securio, 100 sex' frumenti, 450 sex' silginis, 450 sex' avene.

66 Item tradidit idem Aymericus, de mandato domini Oliverii de Cloyquin et Guillelmi de Rogeyo, reformatorum, a dicto festo beate Lucie usque ad sequens festo Purificacionis beate Marie ${ }^{93} 26$ hominibus armorum custodentibus dictum castrum pro suis victualibus, viz., omnibus ipsorum 10 sex' siliginis, 6 sex' avene, valent 260 sex' siliginis, 156 sex' avene.

67 Item tradidit Petrus Fulcherii, ad exoneracionem dicti Aymerici, dicto domino de Porta pro facto guerre dicti vicecomitis usque ad 1500 sex' bladorum siliginis vel circa et de residuo firme sue de Payzaco satisfecit dicto Aymerico ad opus victualibus gentorum armorum dicti castri de Securio ad complend' dictorum saumiarum (?) ipsis gentibus per dictum Aymericum ut predicitur traditam.

Summa universalis omnium solucionum et misiarum bladorum predictorum computis solucionibus pro redditibus factis 3416 sext'

68 Et sit de blado dicte annate potuerit restare 331 sex' mosdinarum quas mosduras dictis receptor dicitur vendidisse assensacoribus dictorum molendinorum.

69 Recepta vini rendualis, 22 saumatas, viz. 10 saumatas vini albi et 12 saumatas vini rubei quod vinum adhuc debetur en essaudoues.

70 Recepta cere, 90 libras cere.

91. Vers le 15 août 1345 ou 1346 ?

92. 8 septembre-13 décembre 1345 .

93. 13 décembre 1345-2 février 1346. 
71 Recepta feni, 393/4 trossas de Segur et de Payzac de quo dominus de Castro Bocheti fecit levare certam partem.

72 Recepta guallinarum, 650 guall'.

73 Misia dictarum guallinarum, primo, daisivisit idem Aymericus in Secgurio, Santo Perdulphi domino de Porta $\mathrm{XV}^{\mathrm{e}}$ die mensis augusti anno XL quinto, 92 gall'.

74 Item expendit idem dominus de Porta cum comtiva sua pro visitacionem castri de Securio in nativitate beate Marie ${ }^{94}, 20$ gall'.

75 Item expendit dominus Olivierius de Cloyquin et Guillelmus de Rogeyo apud Securio, 13 gall'.

76 Item expendit dominus de Castro Bocheti apud Securio, 8 gall'.

77 Et eciam de restaur' gall' idem dominus de Castro Bocheti fecit levare plurium gall' et adhuc levantur.

78 Recepta cutellarum, 140 cutellas de quibus Helie de Nova Villa habuit 100 et alii capitanei habuere 40 cutellas.

79 Recepta castanearum, ${ }^{95} 4$ sex' ad veterem mensuram.

80 Recepta liquorum, una quadrigata.

Sciendum est quo de tallis que facte fuerunt in castellanie de Securio in anno XL quinto Aymericus de Bona Villa non habunt nec habere potuitur a baiul' dicte castellanie aliquid nisi ab hominibus a quibus prepositus levare consuevit illas $60 \mathrm{l}$. de quibus supra computavit in recepta denarii attande (?) sequentur baiuli dicunt se solvisse de tallis pro eos levatos que sequntur

81 Johannes de Perduris domino de Porta 20 l. et domino Guillelmo Coteti 40 l.

82 Adem' Michael domino de Porta 75 l., dicto Guillelmo Coteti 4 l., Magistro Petro Puihol 20 1., Guido Raffart, domino Gauffrido Helie, $50 \mathrm{l}$.

Et protestatur idem Aymericus de supplendo, concedando, corrigendo, augmentando et diminuendo in et super premissis prout debebit et sint (?) faciendum, et quam premissa non tradit per modum computi in (?) cuiusdam summarie avizeya. 


\section{RÉSUMÉ}

L'administration de la vicomté de Limoges par Arthur II de Bretagne, qui l'avait acquise par mariage en 1275, et par ses successeurs n'a, jusqu'ici, pas été sérieusement étudiée. La découverte de quelques rares témoignages financiers datant du règne de Charles de Blois et de Jeanne de Penthièvre, duc et duchesse de Bretagne (1341-1364) et vicomte et vicomtesse de Limoges à partir de 1343, nous permet d'examiner en détail comment la vicomté était gouvernée et quelles ressources elle offrait à ses souverains. Un compte du receveur de Charles de Blois à Limoges pour la période 1344-1347 et les comptes du prévôt de la châtellenie de Ségur-le-Château pour 1345-1346, qui sont parmi les plus anciens témoignages de ce genre pour la vicomté, illustrent les développements sociaux, économiques et militaires connus par celle-ci alors que Limoges s'impliquait de plus en plus dans la succession contestée du duché de Bretagne (après la mort de Jean III en 1341), mais aussi dans le conflit plus général qui allait engloutir l'ouest de la France dans la première phase de la guerre de Cent Ans. Une édition des comptes de Ségur est annexée à l'étude.

\section{ABSTRACT}

The administration of the viscounty of Limoges by Arthur II of Brittany, who had acquired it by marriage in 1275, and his successors has not been seriously studied. The discovery of some rare financial evidence dating to the reign of Charles de Blois and Jeanne de Penthièvre, duke and duchess of Brittany (1341-1364), and viscount and viscountess of Limoges from 1343 onwards, allows us to examine in some detail how the viscounty was governed and what resources it offered its rulers. An account of the receiver of Charles de Blois in Limoges for the period 1344-1347 and the accounts of the provost of the castellany of Ségur-le-Château for 1345-1346, amongst the earliest such records surviving for the viscounty, illustrate social, economic and military developments as Limoges became more and more involved not only in the disputed succession to the duchy of Brittany (following the death of John III in 1341) but also the more general conflict that would engulf western France in the first phase of the Hundred Years War. An edition of the Ségur accounts is appended to the study. 